\title{
Comparison of Aspects of Smoking Among Four Histologic Types of Lung Cancer
}

\author{
Stacey A. Kenfield, ScD ${ }^{1,2}$, Esther K. Wei, ScD ${ }^{1,2}$, Meir J. Stampfer, MD, DrPH ${ }^{1,2}$, Bernard \\ A. Rosner, PhD 2,3 , and Graham A. Colditz, MD, DrPH ${ }^{4}$ \\ ${ }^{1}$ Department of Epidemiology, Harvard School of Public Health, Boston, MA, USA \\ ${ }^{2}$ Channing Laboratory, Department of Medicine, Brigham and Women's Hospital and Harvard \\ Medical School, Boston, MA, USA \\ ${ }^{3}$ Department of Biostatistics, Harvard School of Public Health, Boston, MA, USA \\ ${ }^{4}$ Department of Surgery, Washington University School of Medicine, St. Louis, MO, USA
}

\begin{abstract}
Background-The magnitude of the link between cigarette smoking and lung cancer may vary by histologic type.
\end{abstract}

Methods-We used polytomous logistic regression to evaluate whether aspects of smoking have different effects across four histologic types in the Nurses' Health Study.

Results-From 1976 through 2002, we identified 1,062 cases of lung cancer: squamous cell $(n=201)$, small cell $(n=236)$, adenocarcinoma $(n=543)$, and large cell carcinoma $(n=82)$, among 65,560 current or former smokers. Risk reduction after quitting ranged from an $8 \%$ reduction (relative risk (RR): $0.92,95 \%$ confidence interval $(\mathrm{CI}): 0.91,0.94)$ to a $17 \%$ reduction (RR: 0.83 , $95 \%$ CI: $0.80,0.86)$ per year for adenocarcinoma and small cell carcinoma, respectively, with a $9 \%$ reduction observed for large cell carcinoma and an $11 \%$ reduction observed for squamous cell carcinoma. The association of age at smoking initiation and former cigarette smoking was similar across types, while the association of smoking duration differed. The risk of adenocarcinoma increased by $6 \%$ per year of smoking, compared to $7 \%$ for large cell, $10 \%$ for squamous cell, and $12 \%$ for small cell. The $6 \%$ difference between adenocarcinoma and small cell carcinoma is equivalent to a 3.2 to 9.7 -fold increase in risk for 20 years of smoking.

Conclusions-The effects of the number of cigarettes smoked per day and years since quitting smoking are different across the major types of lung cancer, which are fully appreciated at long durations of smoking and smoking cessation. Smoking prevention and cessation should continue to be the focus of public health efforts to reduce lung cancer incidence and mortality.

\section{Keywords}

smoking and smoking cessation; cancer prevention; lung cancer

Smoking increases risk of all major histologic types of lung cancer, a finding that has been recognized since the 1980 US Surgeon General's Report. ${ }^{1}$ The data for women, mostly from case-control studies, indicate that the risk of all histologic types of lung cancer is higher in smokers than nonsmokers, higher in current than former smokers, increases with the quantity and duration of smoking, and is reduced after smoking cessation, ${ }^{2}$ with relative 
risks for current smoking highest in small cell carcinoma, followed by squamous cell carcinoma, and lowest in adenocarcinoma. ${ }^{3-5}$

Case-control studies of smoking and lung cancer are vulnerable to recall bias; some studies rely on smoking information from medical records or next of kin, which provide a limited assessment of smoking history. Cohort studies among women that evaluate smoking and risk of lung cancer classified according to histologic type tend to have few cases, yielding modest statistical power, and can therefore evaluate only a subset of the main histologic types or must combine types. ${ }^{6,7}$ In addition, comparisons between histologic types are limited further by the small number of participants who are non-smokers, leading to unstable risk estimates when these non-smokers are used as the reference group.

We, therefore, formally evaluated whether various aspects of smoking have significantly different effects across the main histologic types of lung cancer. To further understand which aspects convey different risks by histologic type, we used polytomous logistic regression techniques in the Nurses' Health Study to compare risk estimates for small cell carcinoma, squamous cell carcinoma, adenocarcinoma, and large cell carcinoma.

\section{METHODS}

\section{Study participants}

The Nurses' Health Study (NHS) cohort was established in 1976 when 121,700 female US registered nurses between the ages of 30 and 55 years residing in 11 states completed a mailed questionnaire. Follow-up of the original cohort has been greater than $94 \%$ through 2002. ${ }^{8}$ The institutional review board of Brigham and Women's Hospital in Boston approved this study. Participants provided detailed information about medical history and risk factors for cancer at baseline including current and former smoking habits, and every two years since 1976 this information has been updated and extended on follow-up questionnaires. We excluded from our analysis participants with a prior history of cancer (other than non-melanoma skin cancer) at baseline. In preliminary analyses, we used neversmokers $(n=51,088)$ as the referent group. For the polytomous logistic regression analysis, we only included current $(n=37,990)$ or former smokers $(n=26,427)$ at baseline or those who become smokers during the follow-up period $(\mathrm{n}=1,143)$, and excluded those with unknown age at starting $(\mathrm{n}=1,061)$, leaving 65,560 women in this analysis. Current and former smokers contributed approximately 552,000 and 879,000 person-years, respectively, to this analysis.

\section{Assessment of smoking}

On the initial 1976 questionnaire, participants reported whether they currently smoked or had ever smoked in the past, and the age they started smoking. Former smokers reported their age at which they stopped smoking and the number of cigarettes smoked/day before quitting. Current smokers reported the number of cigarettes typically smoked/day. On each subsequent biennial questionnaire, participants reported whether they currently smoke cigarettes, and if so, the number of cigarettes smoked/day in specified categories (1-4, 5-14, $15-24,25-34,35-44$, and $\geq 45$ ). To reduce misclassification, participants were re-classified at the start of each two-year follow-up cycle by smoking status (never, former, or current), by quantity of cigarettes smoked and duration among current smokers, and by time since quitting among former smokers. If no follow-up questionnaire was returned, the most recent record of exposure status was carried forward for the subsequent interval. If a participant failed to return two consecutive questionnaires, smoking status was reclassified as follows: current smokers were classified as missing, former smokers who quit for $>10$ years retained 
their last exposure status, and former smokers who quit for $<10$ years were classified as missing (recent quitters may not remain lifelong quitters).

\section{Ascertainment of lung cancer by histologic type}

Whenever a participant reports a lung cancer, we seek the medical record and pathology reports to confirm the diagnosis and histology. Deaths are usually reported by families, and deaths among non-respondents are identified by searching the National Death Index; very few deaths $(<2 \%)$ are missed. ${ }^{9}$ We ascertain the cause of death and seek the pertinent medical records. For lung cancer cases identified from death reports, we also sought medical records. Study physicians review these records and classify lung cancer cases by histologic type. Physicians were not aware of the participant's smoking history as reported on NHS questionnaires. Cases were considered confirmed only if a pathology report indicated that the lesion was a primary lung tumor. Less than $3 \%$ of reported lung cancers were rejected after medical record review or from insufficient clinical information. During follow-up of 65,560 women from 1976 through June 2002, we confirmed 201 squamous cell, 236 small cell, 543 adenocarcinoma, and 82 large cell carcinoma cases.

\section{Covariates}

BMI $\left(\mathrm{kg} / \mathrm{m}^{2}\right)$, physical activity, and dietary intake of alcohol, $\alpha$-carotene, $\beta$-cryptoxanthin, and lycopene were assessed as confounders of the smoking-histologic type of lung cancer relation. BMI was calculated using height, reported on the 1976 questionnaire, and body weight, which is updated with each biennial questionnaire. Dietary intake was assessed every two to four years since 1980 with a food frequency questionnaire. Physical activity was also assessed repeatedly since the 1980 questionnaire. Participants reported whether they engaged in exercise intensely enough to produce sweat, and if so, the intensity level and specific activity they engaged in most frequently. Since 1986, physical activity was calculated using metabolic equivalent task [MET] hours/week for each participant and is updated biennially.

\section{Statistical analysis}

Each study participant contributed person-time from the date of return of the 1976 questionnaire to the date of a lung cancer diagnosis, death from any cause, or to May 31, 2002, whichever came first. Separate logistic regression models were used to estimate relative risks for cigarettes smoked/day for current smokers and years since quitting for former smokers, using never-smokers as the referent group, for comparison with the existing literature. We used pooled logistic regression, where each 2-year set of observations for each subject is pooled in the logistic regression analysis. Only 105 cases of lung cancer occurred in never-smokers: 7 squamous cell carcinoma, 2 small cell carcinoma, 85 adenocarcinoma, and 11 large cell carcinoma cases. Polytomous logistic regression (PLR) was used to evaluate the relation between various aspects of smoking and risk of four histologic types of lung cancer. A description of the method and its application have been published previously. 10 For this study a custom software program developed and described by Marshall and Chisholm was used, 11 which provides formal tests of the differences in magnitude of the estimate for the coefficient of each risk factor for the separate components of a composite endpoint. In this analysis, our composite endpoint was the four main histologic types of lung cancer. This method provides flexibility in allowing some variables to be modeled with a common beta estimate for all outcomes, either based on a priori hypotheses or through a stepwise procedure based on likelihood ratio tests (discussed below), and some variables to be modeled with four distinct beta estimates, which allows appropriate modeling of all variables. 
We began with a baseline model that allowed all of the risk estimates to vary for all exposure variables between each of the four outcomes. Given $\beta_{1 \text {,small }}$ (the effect of

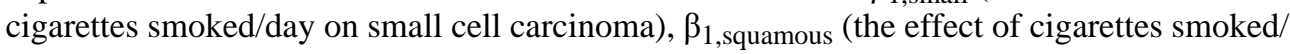

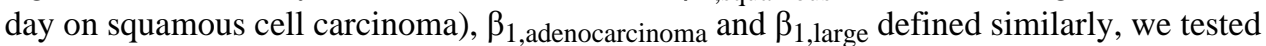
the null hypothesis $\left(\mathrm{H}_{0}: \beta_{1, \mathrm{sm}}=\beta_{1, \mathrm{sq}}=\beta_{1, \mathrm{ad}}=\beta_{1, \mathrm{lg}}\right)$ versus $\mathrm{H}_{1}$ : the effect of cigarettes smoked/day is different on at least two histologic types. Under either $\mathrm{H}_{0}$ or $\mathrm{H}_{1}$, the effect of all the other risk factors in the model is assumed to be different among the four histologic types. A similar test was performed for all other risk factors in the model. We then conducted a step-wise down procedure, whereby each model was tested with the baseline model. Using log-likelihood analyses, the variable with the highest p-value was set to be the same for all outcomes, indicating we could not reject the null of the effects being the same. This became the new baseline model for the next set of tests, and this procedure was repeated, setting more variables to be the same until the remaining variables had p-values less than or equal to 0.05 , indicating they were likely to have different associations with the four outcomes. P-values were computed based on likelihood ratio tests comparing models where the variable was assumed to have a different versus similar effect on each histologic type. Statistical tests were two-sided.

Modeling five outcome categories (non-diseased and each of the four histologic types of lung cancer) is extremely computationally intensive. To reduce the complexity of the computations, we used continuous variables for all smoking-related factors. For cigarettes smoked/day and time since quitting smoking we used the medians of the categories used in the questionnaires. We calculated cigarettes smoked/day using a cumulative average of smoking dose. Specification of the variables in the models was carefully considered to prevent over-adjustment for the time-related dimensions of smoking history and age. For example, current age, age at start of smoking, time since quitting and smoking duration are interdependent and specification of any three variables will determine the fourth. Model 1 included cigarettes smoked/day (cumulative average) for current and former smokers, age at start of smoking, time since quitting smoking, and age. Model 2 included cigarettes smoked/ day (cumulative average) for current and former smokers, smoking duration, and age.

The effect of age on lung cancer risk was evaluated by two methods: 1) modeling age as a continuous variable and 2) modeling age using the following parameters: $\beta_{1}$ (min ((current age, age at menopause) $)+\beta_{2}$ (current age-age at menopause)*menopausal status. This second method was tested as the age incidence curve for lung cancer is not linear across ages; the slope of the age incidence curve becomes steeper approximately at the time of menopause. ${ }^{12}$ Therefore, this latter method of evaluating the effect of age may be more precise than modeling age as a continuous variable.

\section{RESULTS}

Table 1 shows the characteristics of the study population across the outcome categories. Compared to the other histologic types, a larger percentage of participants who developed adenocarcinoma were former smokers at baseline in 1976 and before diagnosis (16\% and $48 \%$, respectively). In contrast, those developing small cell carcinoma were mainly current smokers in 1976 and before diagnosis (96\% and 75\%). A larger proportion of squamous cell carcinoma and adenocarcinoma cases smoked less than 15 cigarettes per day in 1976, compared to those developing small cell and large cell carcinoma. More than half of the women diagnosed with squamous cell, small cell, or adenocarcinoma started smoking between ages 15 and 19, whereas almost half of those with large cell carcinoma started smoking at age 20 or later. 
Compared to never smokers, relative risks for the lowest smoking duration ( 1 to $<20$, or 20 to $<30$ years) ranged from 0.83 for adenocarcinoma to 8.59 for small cell carcinoma and for the highest smoking intensity (40+ years) ranged from 3.57 for large cell carcinoma to 77.0 for small cell carcinoma (Table 2). Relative risks for former smoking were higher for small cell carcinoma than for the other histologic types for 1 to 20 years since quitting, with the excess risk for each histologic type decreasing to the level of a never-smoker after 20 years (Table 3). Despite the size of this cohort, some of the confidence intervals are quite wide since never-smokers served as the reference category and very few cases of small cell, squamous cell, and large cell carcinoma occurred in this group.

Risk estimates from the final models are presented in Table 4a and 5a. Based on the Marshall and Chisholm method, the first model indicated that years since quitting and age have significantly different risk estimates for the different histologic types of lung cancer (Table 4a). The risk for small cell carcinoma declined by $17 \%$ per year of smoking cessation (relative risk (RR): 0.83 ; 95\% confidence interval (CI): 0.80, 0.86), while the corresponding decline for adenocarcinoma was $8 \%$ per year (RR: $0.92 ; 95 \%$ CI: $0.91,0.94)$. These decreases in risk are equivalent to a $94 \%$ reduction for risk of small cell carcinoma and $70 \%$ reduction for adenocarcinoma after 15 years of quitting smoking (Table $4 \mathrm{~b}$ ). We obtained the same results using age modeled as continuous or using age at menopause and menopausal status (as discussed above). The risk estimates from the simpler model are therefore presented in Table 4a and 5a. The association of age at smoking initiation and cigarettes smoked/day for current and former smokers did not differ significantly between the histologic types in Model 1 (Table 4a); however, in Model 2, there was a small difference in the effect of cigarettes smoked/day for current smokers across histologic types (Table 5a). The relative risk was 1.07 for small cell carcinoma, 1.06 for large cell carcinoma, and 1.05 for the other types, equivalent to a 3.87-fold, 3.21-fold, and 2.65-fold increase in the risk of these types, respectively, per pack smoked. There was a different and considerable effect of smoking duration across the histologic types, with the risk of adenocarcinoma increasing by $6 \%$ per year of smoking compared to $12 \%$ per year for small cell carcinoma (Table 5a). These increases in risk are equivalent to a 2.45 to 5.14-fold increase for 15 years of cigarette smoking (Table 5b). Controlling for BMI, physical activity, and alcohol, $\alpha$-carotene, $\beta$-cryptoxanthin, and lycopene intake resulted in minimal change in effect estimates, and therefore these variables were not included in our final PLR model.

\section{DISCUSSION}

Our prospective analysis indicates that among women, there is a different effect of smoking dose across histologic types of lung cancer, with greater variation in risk for smoking duration than for the number of cigarettes smoked/day. Upon cessation, the risk for small cell carcinoma drops more rapidly than the other histologic types.

Quitting smoking clearly reduces the risk of developing any type of lung cancer, compared with continuing. Our findings showing the decline in risk after quitting is strongest for small cell carcinoma (followed by squamous cell carcinoma, large cell carcinoma, and adenocarcinoma, respectively), is supported by previous studies assessing the relation of cessation and risk of lung cancer by histologic type, as well as those studies indicating that this histologic type is most associated with smoking. ${ }^{7} 13$ This finding is meaningful as small cell carcinoma of the lung has the most aggressive clinical course of all pulmonary tumors, with a median survival of 16-24 months with current forms of treatment for patients with limited-stage disease. $14^{-} 16$ 
The strength of the association with tobacco smoke may differ by histologic type due to lower exposure of tobacco smoke particles to sites that are more peripheral in the respiratory tract. Squamous and small cell carcinoma occur mainly in the large central bronchi, an area highly exposed to tobacco smoke, compared to adenocarcinoma, located in the peripheral sections of the lung, and large cell carcinoma, located in the peripheral and subpleural regions.17 Although cigarette design changes and smoking behavior may have allowed adenocarcinoma to become more strongly associated with cigarette smoking than it has been in the past, through an increase in the dose of potent tobacco-specific nitrosamine 4(methylnitrosamino)-1-(3-pyridyl)-1-butanone (NNK)18,19 and smoker compensation (more frequent puffs per cigarette, larger puff volumes, deeper inhalation),20 the effect of smoking duration still has a substantially different effect across histologic types compared to the number of cigarettes smoked/day. Our finding that smoking duration is a more important predictor than smoking intensity for lung cancer, is supported by two large studies,21,22 and this duration effect may account for most of the difference in the effect of smoking between histologic types, obscured when using pack-years.23,24

Some other aspects of smoking do not seem to have different effects on the histologic types, such as age at starting smoking. However, as $63 \%$ of our cohort began smoking between 18 and 21 years of age, and less than $5 \%$ started at 30 years of age or later, there may not have been enough variability to detect a different effect of age at starting smoking according to histologic type. Our results also indicated that the association between cigarettes smoked/ day by former smokers and risk of the four histologic types are similar when modeled as continuous variables. Former smokers who quit smoking before 1976 only provided information on the amount of cigarettes smoked before quitting; thus, we cannot fully capture the smoking history of a former smoker, which may prevent us from precisely estimating the risks associated with smoking intensity for this group.

Five recent prospective cohort studies examined cigarette smoking and subsequent risk of lung cancer by histologic type, although none formally compared risk estimates across the histologic types. Among current male smokers in the Japanese Public Health Center Study, the magnitude of relative risk was greater for smoking duration than the number of cigarettes smoked/day when modeling these variables simultaneously, similar to our results, and a more rapid increase in risk with increasing duration was observed for squamous cell and small cell carcinomas combined $\left(\mathrm{n}=101 ; P_{\text {trend, duration }}=0.06\right)$, compared to adenocarcinoma $\left(\mathrm{n}=78 ; P_{\text {trend, duration }}=0.03\right){ }^{25}$ In the Norwegian male cohort, a more rapid increase in risk with increasing cigarettes per day was observed for small cell $(\mathrm{n}=45)$ and squamous cell carcinoma ( $n=99)$, compared with adenocarcinoma $(n=42) .{ }^{26}$ Both studies had too few cases in women to provide separate estimates by histologic type and only had smoking information reported by participants at baseline.

Among current male smokers in the Korean 24 and Japanese studies, 25 and female and male smokers in the Copenhagen cohort, ${ }^{27}$ risk increased with higher pack-years for adenocarcinoma, squamous cell carcinoma and small cell carcinoma, but a steeper increase was observed for squamous and small cell carcinoma. In the Iowa Women's Health Study, there was a more consistent risk reduction with smoking cessation for squamous/small cell carcinoma than with adenocarcinoma. Compared with current heavier smokers ( $\geq 20$ packyears), there was a significantly lower risk of squamous/small cell carcinoma $(n=39)$ but a non-significantly lower risk of adenocarcinoma $(n=31)$ among former heavier smokers in the first 10 years of smoking cessation. ${ }^{7}$ None of these studies evaluated large cell carcinoma.

In our study, repeated measures on smoking status collected every two years have mitigated misclassification due to changes in smoking status over time. Follow-up is very high in the Nurses' Health Study and there are high rates of histologic confirmation of primary lung 
cancer. The accuracy of the classification of lung cancer by histologic type may not be perfectly consistent. However, as standard diagnostic criteria are applied, the classifications of cell type are likely to be reasonably accurate. One study compared reports to the Iowa Cancer Registry with an independent uniform review by 2 board certified surgical pathologists, and found that the histologic type diagnosis obtained by the registry was reasonably reliable, with an overall percent exact agreement of $72 \%{ }^{28}$ Small cell carcinoma had the highest sensitivity (94.1\%) while large cell carcinoma had the lowest sensitivity (21.9\%), explained in part by the absence of specific features that would permit a diagnosis of adenocarcinoma or squamous carcinoma. Specificity was lowest for adenocarcinoma (84.4\%). Inaccuracy in the classification of histologic type may prevent us from observing a difference in risk for the various smoking factors across the four histologic types of lung cancer.

Depth of inhalation and tar content were not accounted for in this analysis. Data on tar is not available in this cohort; however, there is evidence that compensatory changes are observed with "reduced yield" cigarettes, such as increasing the number of cigarettes smoked/day, reducing the benefit of lower-tar cigarettes and potential for residual confounding by tar content. ${ }^{29,30} \mathrm{We}$ only had data on depth of inhalation for current smokers, and this aspect was only queried on two early questionnaires, so we could not account for changes in smoking inhalation behavior over follow-up. As most adenocarcinoma occurs in the periphery of the lung, which could indicate deeper inhalation, there may be different effects of inhalation across the histologic types. The data on inhalation in the study by Prescott et al. suggest that inhalation may modify the effect of pack-years, showing that an increase in risk of lung cancer with increasing pack-years is stronger for inhalers compared to non-inhalers. ${ }^{27} \mathrm{We}$ did not include exposure to passive smoke in this analysis; however, passive smoke exposure is unlikely to be an important factor among ever smokers.

In summary, our findings indicate that smoking is a cause of all major types of lung cancer in women, with the risk of small cell and squamous cell carcinoma increasing most rapidly with increasing smoking duration. The risk of all types of lung cancer examined decreases after quitting, with risk of small cell carcinoma decreasing most rapidly after cessation. These results are fully appreciated at long durations of smoking and smoking cessation. Preventing smoking initiation and encouraging smoking cessation should continue to be the focus of public health efforts to reduce lung cancer incidence and mortality.

\section{"What this paper adds"}

Smoking increases risk of all major histologic types of lung cancer. Case-control and cohort studies have previously looked at this association across histologic type, but have not used formal statistical tests to evaluate heterogeneity. Due to a limited number of cases, some cohort studies combined histologic types or did not perform histologic analyses in women. Utilizing repeated measures of smoking status, and the high followup rates in the Nurses' Health Study, this study is the largest cohort study to evaluate the relation between aspects of smoking and risk of lung cancer by histologic type in women, while applying new statistical techniques.

Our prospective analysis indicates that among women, there is a different effect of smoking dose across histologic types of lung cancer, with greater variation in risk for smoking duration than for the number of cigarettes smoked per day. Upon cessation, the risk for small cell carcinoma drops more rapidly than the other histologic types. These different results by histologic type are fully appreciated at long durations of smoking and smoking cessation. Preventing smoking initiation and encouraging smoking cessation 
should continue to be the focus of public health efforts to reduce lung cancer incidence and mortality.

\section{Acknowledgments}

Funding: The research for this article was funded by grant CA87969 and T32CA09001 from the National Institutes of Health, the Association of Schools of Public Health (ASPH), and the Legacy Foundation. The content is solely the responsibility of the authors and does not necessarily represent the views of the National Cancer Institute of the National Institutes of Health, ASPH, Legacy, Legacy Foundation staff, or Legacy's Board of Directors.

\section{REFERENCES}

1. US Department of Health and Human Services. The health consequences of smoking for women: a report of the Surgeon General. Washington, DC: U.S. Dept of Health and Human Services, Public Health Service, Office of the Assistant Secretary for Health, Office on Smoking and Health; 1980. p. 109-131.

2. US Department of Health and Human Services. Women and smoking: a report of the Surgeon General. Rockville (MD): U.S. Dept of Health and Human Services, Public Health Service, Office of the Surgeon General; 2001. p. 177-449.

3. Schoenberg JB, Wilcox HB, Mason TJ, et al. Variation in smoking-related risk among New Jersey women. Am J Epidemiol 1989;130:688-95. [PubMed: 2549787]

4. Brownson RC, Chang JC, Davis JR. Gender and histologic type variations in smoking-related risk of lung cancer. Epidemiology 1992b;3:61-4. [PubMed: 1313311]

5. Osann KE, Anton-Culver H, Kurosaki T, et al. Sex differences in lung cancer risk associated with cigarette smoking. Int J Cancer 1993;54:44-8. [PubMed: 8386708]

6. Sobue T, Yamamoto S, Hara M, et al. Cigarette smoking and subsequent risk of lung cancer by histologic type in middle-aged Japanese men and women: the JPHC study. Int J. Cancer 2002;99:245-251. [PubMed: 11979440]

7. Ebbert JO, Yang P, Vachon CM, et al. Lung cancer risk reduction after smoking cessation: observations from a prospective cohort of women. J Clin Oncol 2003;21:921-6. [PubMed: 12610194]

8. Colditz GA, Manson JE, Hankinson SE. The Nurses' Health Study: 20-year contribution to the understanding of health among women. J Womens Health 1997;6:49-62. [PubMed: 9065374]

9. Rich-Edwards J, Corsano K, Stampfer MJ. Test of the National Death Index and Equifax Nationwide Death Search. Am J Epidemiol 1994;140:1016-9. [PubMed: 7985649]

10. Glynn RJ, Rosner B. Methods to evaluate risks for composite end points and their individual components. J Clin Epidemiol 2004;57:113-22. [PubMed: 15125620]

11. Marshall R, Chisholm E. Hypothesis testing in the polychotomous logistic regression model with an application to detecting gastrointestinal cancer. Stat Med 1985;4:337-344. [PubMed: 4059720]

12. Bain C, Feskanich D, Speizer FE, et al. Lung Cancer Rates in Men and Women with Comparable Histories of Smoking. J Natl Cancer Inst 2004;96:826-34. [PubMed: 15173266]

13. Khuder SA, Mutgi AB. Effect of Smoking Cessation on Major Histologic Types of Lung Cancer. Chest 2001;120:1577-1583. [PubMed: 11713137]

14. Murray N, Coy P, Pater JL, et al. Importance of timing for thoracic irradiation in the combined modality treatment of limited-stage small-cell lung cancer. The National Cancer Institute of Canada Clinical Trials Group. J Clin Oncol 1993;11:336-44. [PubMed: 8381164]

15. Johnson BE, Bridges JD, Sobczeck M, et al. Patients with limited-stage small-cell lung cancer treated with concurrent twice-daily chest radiotherapy and etoposide/cisplatin followed by cyclophosphamide, doxorubicin, and vincristine. J Clin Oncol 1996;14:806-13. [PubMed: 8622028]

16. Turrisi AT 3rd, Kim K, Blum R, et al. Twice-daily compared with once-daily thoracic radiotherapy in limited small-cell lung cancer treated concurrently with cisplatin and etoposide. N Engl J Med 1999;340:265-71. [PubMed: 9920950] 
17. Morabia A, Wynder EL. Cigarette Smoking and Lung Cancer Cell Types. Cancer 1991;68:20742078. [PubMed: 1655236]

18. Hoffmann D, Hoffmann I. The changing cigarette, 1950-1995. J Toxicol Environ Health 1997;50:307-64. [PubMed: 9120872]

19. Hecht SS. Tobacco smoke carcinogens and lung cancer. J Natl Cancer Inst 1999;91:1194-1210. [PubMed: 10413421]

20. Kozlowski, LT.; O’Connor, RJ.; Sweeney, CT. Shopland, DR., editor. Risks associated with smoking cigarettes with low machine-measured yields of tar and nicotine. Smoking and Tobacco Control Monograph No. 13. NIH Pub. No. 02-5074. Bethesda, MD: U.S. Department of Health and Human Services, National Cancer Institute; 2001. Cigarette Design.

21. Doll R, Peto R. Cigarette smoking and bronchial carcinoma: dose and time relationships among regular smokers and lifelong non-smokers. J Epidemiol Community Health 1978;32:303-13. [PubMed: 744822]

22. Flanders WD, Lally CA, Zhu BP, et al. Lung cancer mortality in relation to age, duration of smoking, and daily cigarette consumption: results from Cancer Prevention Study II. Cancer Res 2003;63:6556-62. [PubMed: 14559851]

23. Prescott E, Osler M, Hein HO, et al. Gender and smoking-related risk of lung cancer. The Copenhagen Center for Prospective Population Studies. Epidemiology 1998;9:79-83. [PubMed: 9430273]

24. Yun YH, Lim MK, Jung KW, et al. Relative and absolute risks of cigarette smoking on major histologic types of lung cancer in Korean men. Cancer Epidemiol Biomarkers Prev 2005;14:212530. [PubMed: 16172220]

25. Sobue T, Yamamoto S, Hara M, et al. Cigarette smoking and subsequent risk of lung cancer by histologic type in middle-aged Japanese men and women: the JPHC study. Int J Cancer 2002;99:245-51. [PubMed: 11979440]

26. Engeland A, Haldorsen T, Andersen A, et al. The impact of smoking habits on lung cancer risk: 28 years' observation of 26,000 Norwegian men and women. Cancer Causes Control 1996;7:366376. [PubMed: 8734831]

27. Prescott E, Osler M, Hein HO, et al. Gender and Smoking-Related Risk of Lung Cancer. Epidemiology 1997;9:79-83. [PubMed: 9430273]

28. Field RW, Smith BJ, Platz CE, et al. Lung Cancer Histologic Type in the Surveillance, Epidemiology, and End Results Registry Versus Independent Review. J Natl Cancer Inst 2004;96:1105-1107. [PubMed: 15265973]

29. Thun MJ, Burns DM. Health impact of "reduced yield" cigarettes: a critical assessment of the epidemiological evidence. Tob Control 2001;10(Suppl 1):i4-11. [PubMed: 11740038]

30. Benowitz, NL. Compensatory Smoking of Low-Yield Cigarettes. In: Shopland, DR.; Burns, DM.; Benowitz, NL., et al., editors. Monograph 13: Risks Associated with Smoking Cigarettes with Low Tar Machine-Measured Yields of Tar and Nicotine. National Cancer Institute; Bethesda, MD: 2001. p. 39-63. 


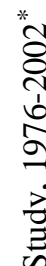

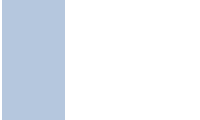

Z 


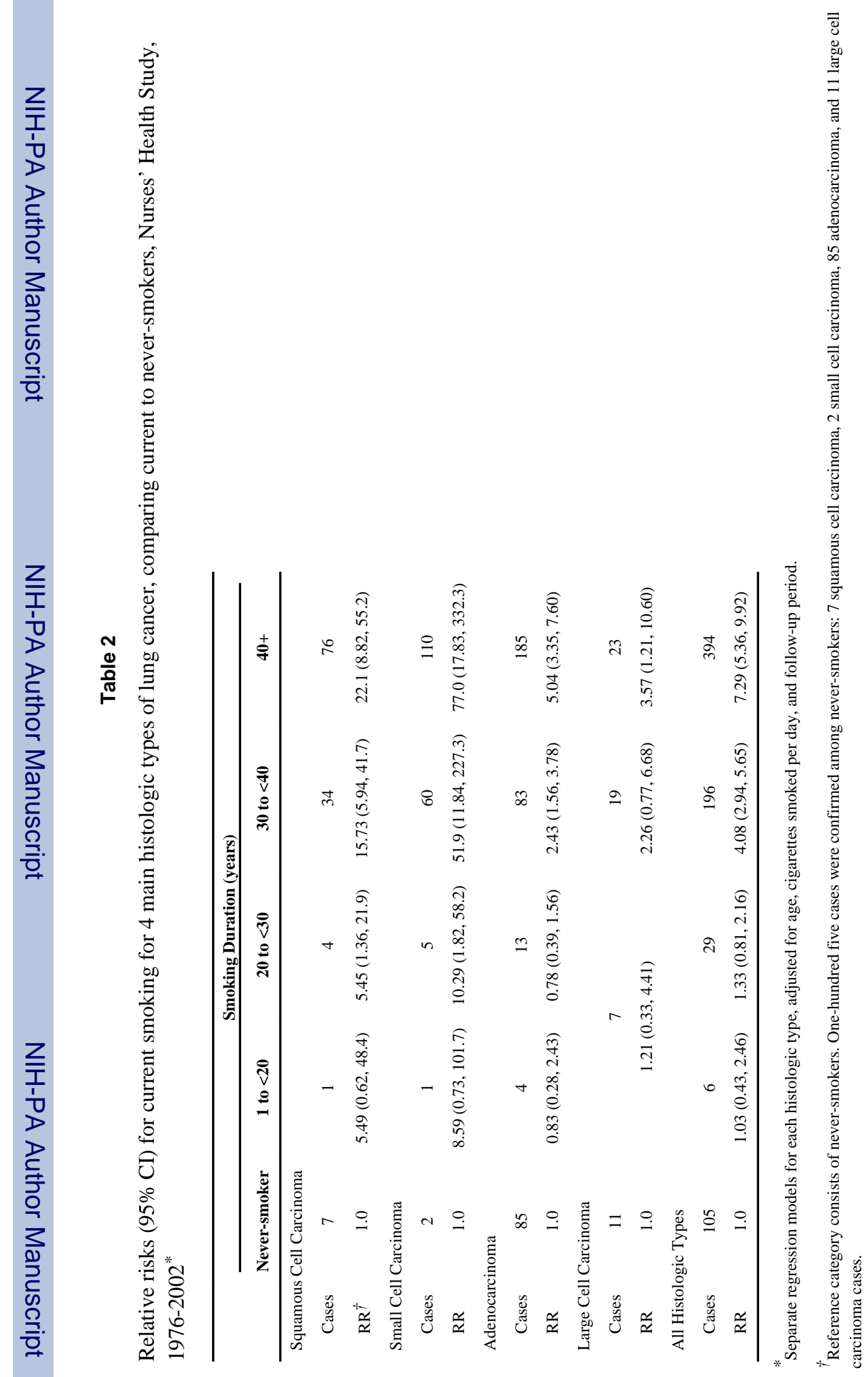




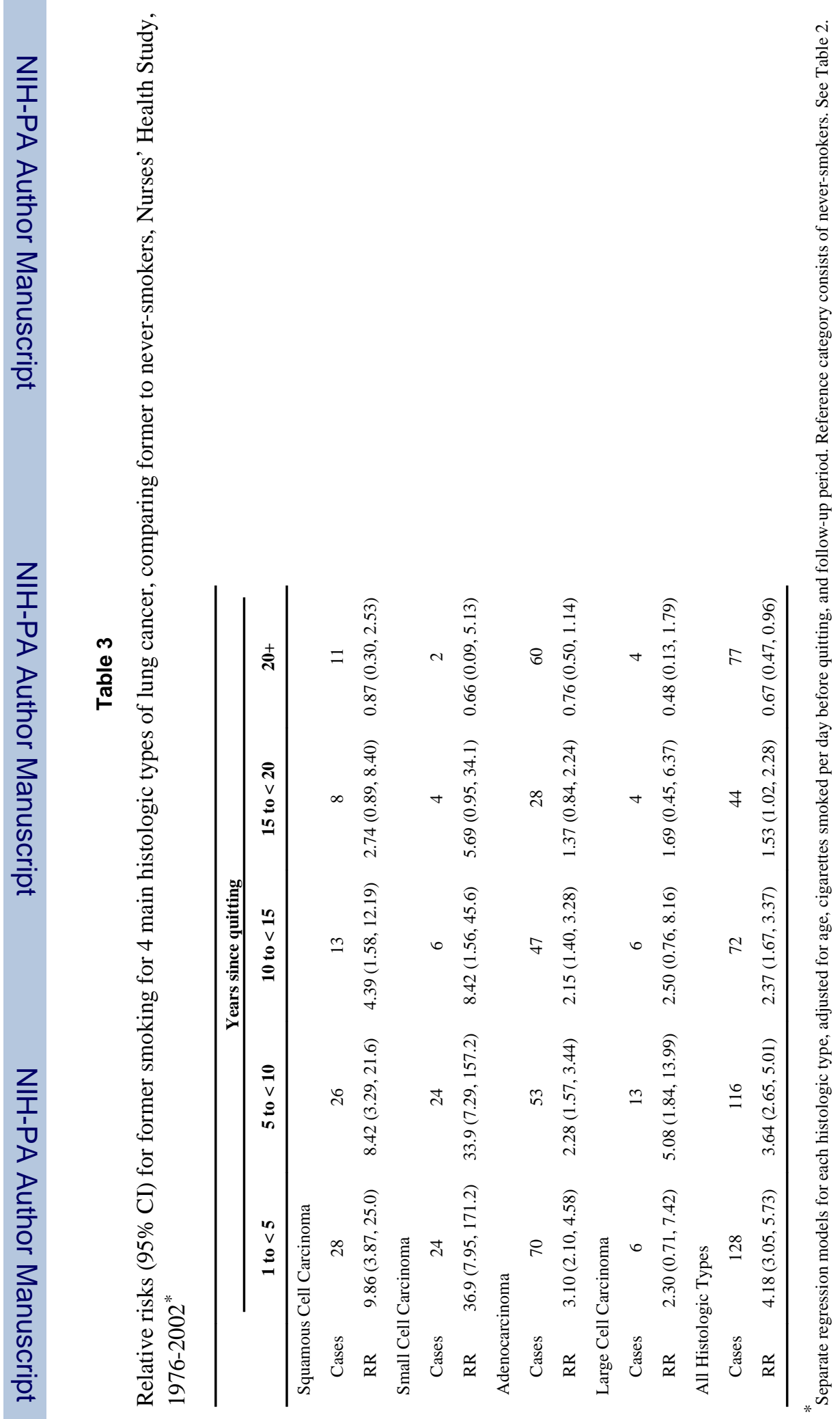




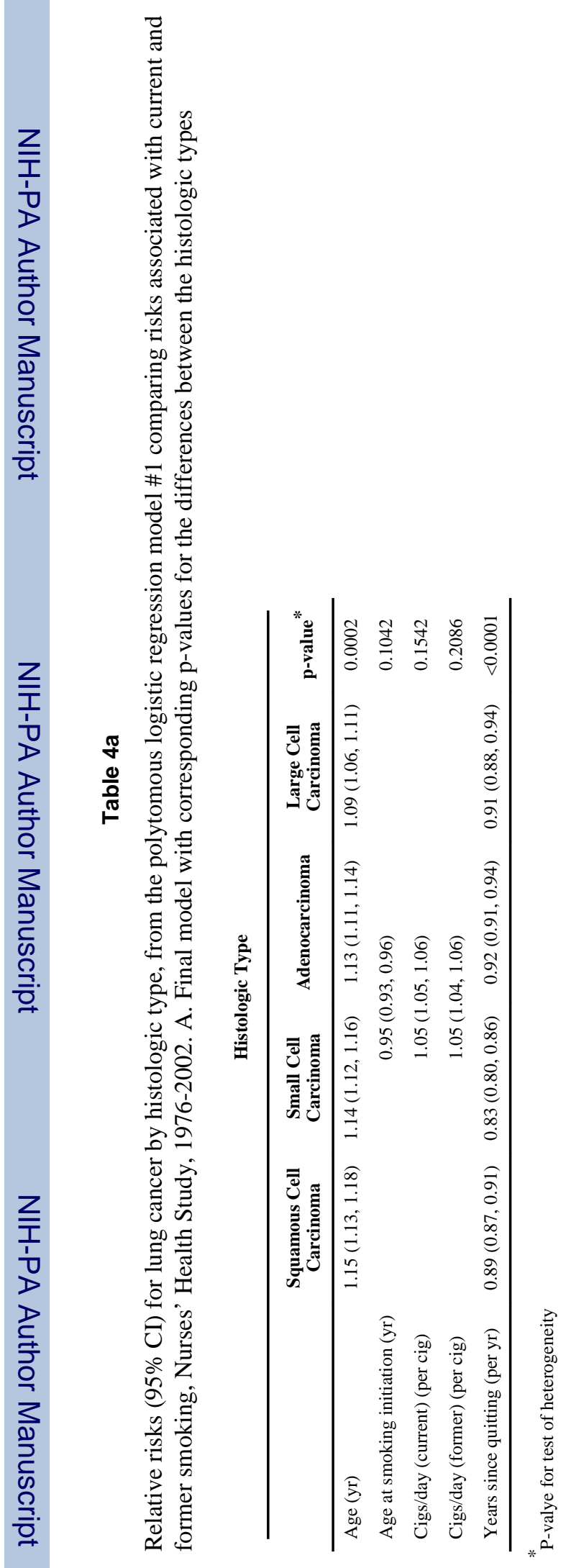




\section{Table 4b}

Relative risks (95\% CI) for lung cancer by histologic type, from the polytomous logistic regression model \#1 comparing risks associated with current and former smoking, Nurses' Health Study, 1976-2002. B. Relative risks corresponding with 5,10 , and 15 years since quitting smoking ${ }^{* *}$

\begin{tabular}{lccc} 
Histologic Type & \multicolumn{3}{c}{ Years Since Quitting } \\
\hline & $\mathbf{5}$ years & 10 years & 15 years \\
\hline Squamous Cell Carcinoma & $0.57(0.50,0.63)$ & $0.32(0.25,0.41)$ & $0.18(0.13,0.26)$ \\
Small Cell Carcinoma & $0.40(0.34,0.47)$ & $0.16(0.11,0.22)$ & $0.06(0.04,0.10)$ \\
Adenocarcinoma & $0.67(0.62,0.72)$ & $0.45(0.39,0.52)$ & $0.30(0.24,0.38)$ \\
Large Cell Carcinoma & $0.63(0.53,0.75)$ & $0.40(0.28,0.56)$ & $0.25(0.15,0.42)$ \\
\hline
\end{tabular}

** Age, age at smoking initiation and cigs/day included in the model 


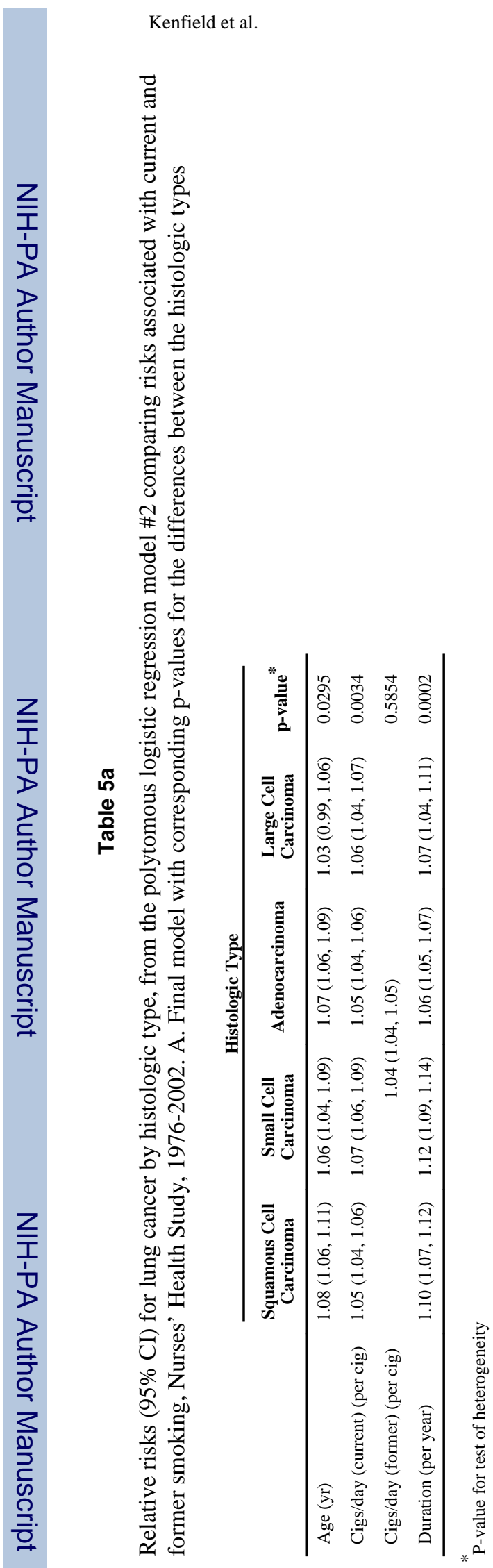

Page 15 


\section{Table 5b}

Relative risks (95\% CI) for lung cancer by histologic type, from the polytomous logistic regression model \#2 comparing risks associated with current and former smoking, Nurses' Health Study, 1976-2002. B. Relative risks corresponding with 5,10 , and 15 years of smoking duration ${ }^{* *}$

\begin{tabular}{lccc} 
Histologic Type & \multicolumn{3}{c}{ Years of Smoking Duration } \\
\hline & 5 years & 10 years & 15 years \\
\hline Squamous Cell Carcinoma & $1.58(1.43,1.75)$ & $2.50(2.04,3.06)$ & $3.95(2.91,5.36)$ \\
Small Cell Carcinoma & $1.73(1.54,1.94)$ & $2.98(2.37,3.74)$ & $5.14(3.64,7.25)$ \\
Adenocarcinoma & $1.35(1.28,1.42)$ & $1.82(1.64,2.02)$ & $2.45(2.10,2.87)$ \\
Large Cell Carcinoma & $1.42(1.23,1.65)$ & $2.02(1.51,2.72)$ & $2.88(1.85,4.49)$ \\
\hline
\end{tabular}

** Age and cigs/day included in the model 